\title{
Beyond Embodiment: From Internal Representation of Action to Symbolic Processes
}

\author{
Isabel Barahona da Fonseca, Jose Barahona da Fonseca, and Vitor Pereira
}

\begin{abstract}
In sensorimotor integration, representation involves an anticipatory model of the action to be performed. This model integrates efferent signals (motor commands), its reafferent consequences (sensory consequences of an organism's own motor action), and other afferences (sensory signals) originated by stimuli independent of the action performed. Representation, a form of internal modeling, is invoked to explain the fact that behavior oriented to the achievement of future goals is relatively independent from the immediate environment. Internal modeling explains how a cognitive system achieves its goals despite variations in the environment with insufficient and noisy sensory-perceptual data. In a self that acts intentionally on the environment, knowledge is dependent upon the necessity to guide actions directed toward an aim. The self-inner model, a representation of internal and external environments (including reafferent and afferent messages) and also of the behavior plans and desirable future states (aims) and efferent intentions (motor planning and motor command messages), is intrinsically linked to a thinking capacity, which is supposed to emerge from the binding of multiple influences. Thinking emerges when higher behavior strategies are considered possible and capable of leading to aims or the fulfillment of intentions. In this model, symbolization processes are projective and anticipatory and, in this way, beyond present referents. Symbolization occurs linked to action planning, command, and regulation in mental simulation. Meaning is related to an inner sense of a self that acts over the environment.
\end{abstract}

I. B. da Fonseca (B)

Department of Psychophysiology, Faculty of Psychology,

Alameda da Universidade, 1613-049 Lisbon, Portugal

Faculty of Psychology, University of Lisbon, Lisbon, Portugal e-mail: isabelbf@fpce.ul.pt

J. B. da Fonseca

Faculty of Sciences and Technology, New University of Lisbon, Lisbon, Portugal

V. Pereira

Faculty of Psychology, University of Lisbon, Lisbon, Portugal

L. Swan (ed.), Origins of Mind, Biosemiotics 8, DOI 10.1007/978-94-007-5419-5_9, (C) Springer

Science+Business Media Dordrecht 2013 
Keywords Cognitive model - Anticipation - Embodiment - Symbolic processes • Neurophysiological functions

\section{Introduction}

The relation between physiological processes and psychic events remains an unsolved problem. It is proposed in this chapter that meaning and symbolic processes are created in an internal space of representation involving the binding between internal and external sensory information, motor command and regulation models, planning of behavior, and anticipation of future aims. Meaning and symbolic processes occur in a projective way linked to efferent processes associated with the planning and command of behavior directed to the external environment and anticipation of future desirable states. From a neurophysiological point of view, these processes occur in a widely distributed network involving cortical and subcortical regions in systems that are massively parallel and interactive (in a neurophysiological sense). The efferent component linked to planning, executive functions, and anticipation depends on prefrontal, cingulate, and parietal networks and also on networks involving basal ganglia.

Another interpretation of the hypothesis that mind is dependent upon an interactive, internal space is the notion that what is represented is a type of information that is not exclusively sensory or motor but involves the interaction between messages of different origins: perceptual, motor programs, and intentions. In other words, a representation emerges in an interactive context in which sensory and motor events are submitted to a compatible frame of coordinates that allows for the creation of internal organism-environment models that are endowed with intention and meaning. For an organism that interacts with the environment, it is crucial to have an internal model at the neuronal level that represents the internal and external environment, and also future desirable states. This model is embodied, as far as it guides the purposeful action of the organism, and independent from physical immediate constraints - although the properties of the environment are represented in a way that is somehow homomorphic with physical constraints, at least in the functional way that allows for adaptive and successful behavior.

The mind builds an internal functional space that represents the characteristics of the external and internal environments as they occur in perception. For an organism to be able to successfully interact with its external environment, perception functions by identifying invariants that are further translated in the nervous system (NS) into well-executed motor actions that delivered back into the external world (Llinas 2001) or in cognitions that are not expressed in motor actions.

For an organism that behaves and moves, taking into account the constraints from the external environment, the distinct properties of its internal space and the properties of the external world should have a continuity in which the coordinates of the external world are translated (transduced) in the internal functional space, preserving homomorphic continuity (Llinas 1987, 2001).

Fonseca, Isabel B; Fonseca, Jose B; Pereira, Vitor. (2013).

Beyond Embodiment: From Internal Representation of Action to Symbolic Processes. Liz Swan (ed.), Origins of Mind, Biosemiotics, Vol. 8, 187-199.

https://doi.org/10.1007/978-94-007-5419-5 9

Springer, Dordrecht.

This manuscript version is made available under the CC-BY-NC-ND 4.0 license

http://creativecommons.org/licenses/by-nc-nd/4.0/ 


\section{The Self as an Agent and the Formation of an Internal Model that Allows Symbolic Processes}

When looking for the hypothetical origins of the symbols utilized in the processes involved in the planning, execution, and regulation of an organism's movement, meaning is created by a sense of agency experienced by the organism. This self integrates multiple influences, sensory and motor, in an anticipatory model of the action planning, an internal simulation, which includes also motor plans and desirable future states that direct decision-making and behavioral planning.

The concept of self (a proto-self or core self) corresponds to the binding of diverse sensorimotor transformations into a single, internal representational model crucial for symbolic processes.

Such anticipation is a fundamental function of the NS: to the organism, especially as regards its adaptation to the environment, what is interesting is what is going to happen in the future, not what has already happened. Past experiences have been memorized and integrated in the internal model and are automatically considered in the mental simulation of an action. This classical hypothesis has been formulated in modem science by Llinas (1987, 2001) who has proposed that thinking capacity emerges from movement internalization. In other words, thinking emerges when higher behavior strategies are considered in terms of potentially leading to the fulfillment of intentions. Movement is related not only to body parts but also to objects from the external world, perceptions, and complex ideas.

For Llinas (2001), if we were able to study action internalization, perhaps we would be able to understand something about our nature - the way we think, learn, and represent ourselves in a self-composite and complex manner.

\section{The Brain as Simulator}

The fundamental function of the NS is action planning and regulation. Action regulation achieved in low-level loops integrates a feedback with an anticipatory component (feed-forward): feedback loops that involve a sensorimotor process of error detection and correction are regulated by feed-forward mechanisms. So feedback and feed-forward loops act on a wide group of synergies that regulate motor primitives. Internal models can be understood as neural mechanisms in the motor systems that reproduce a subset of input/output characteristics, or their inverse. Feed-forward internal models predict sensory consequences from efferent signals (also called corollary discharge) of motor commands issued but not yet executed. Inverse internal models can calculate necessary feed-forward motor commands from a desired final state.

Anticipation is adaptive: it saves time and effort in the execution of a motor program. In anticipation of desired final states, motor programming and execution are independent from a coordinate system, a kind of internal premotor invariance. An example is found in the apparent proficiency, for example, when signing your name using elbow and shoulder joints when writing on a boar, or using any other articulations, such as fingers and hand when signing in a paper, or even foot and leg. The point is that in all these cases, involving such

Fonseca, Isabel B; Fonseca, Jose B; Pereira, Vitor. (2013).

Beyond Embodiment: From Internal Representation of Action to Symbolic Processes. Liz Swan (ed.), Origins of Mind, Biosemiotics, Vol. 8, 187-199.

https://doi.org/10.1007/978-94-007-5419-5_9

Springer, Dordrecht.

This manuscript version is made available under the CC-BY-NC-ND 4.0 license 
diverse articulations, the results of motor executions, the signing, despite different scales and precision, are similar (Llinas 1987). It has been proposed that this constitutes a manifestation of a kind of motor invariance, in which actions are represented in an abstract form associated to the final intended result.

Parallel to this control function, neuronal loops of high level in the motor hierarchy, and also in the phylogenetic scale, begin to be progressively more complex and to function in an anticipatory and projective way. In this projective anticipatory process, brain signals are used to generate action plans (or internal models) in internal loops without direct relation to a present stimulus. The neuronal operations are noncontinuous and occur in neuronal maps whose parameters are the topographical and functional relations between neurons. This mode of prediction about future states, a mental simulation, does a kind of preselection of action strategies and, in general, guides decision-making. In this respect, the brain functions as a simulator projecting future states and strategies.

These processes are foundational for cognitive representation; they integrate and bind multiple signals such as action planning and command. The integration of the meaning of these multiple messages is referred to as a self in the environment, which becomes the center of the phenomenic experience.

\section{The Self as an Agent: The Contribution of Efferent Copy (Efferenze Copie, Von Holst)}

Knowledge is integrated in the internal model, creating conditions that are necessary for symbolic processes to occur within a preconceptual sense of an agent: a proto-self that is nonconscious, but without which, more sophisticated selfexperiences cannot occur. Such functioning that creates an inner cognitive model is linked to the sense of agency-the experience that the subject has of being himself the cause and generation of action (Gallagher 2000, 2012).

Above, we discussed the contribution of the binding of multiple neuronal signals in the creation of the conditions for symbolic processes. These influences are inner sensory experiences related to body (somatosensory signals) and external stimuli (some dependent and others independent from the subject's action), activation of memories of past experiences, action plans, efferent commands, the representation of future desirable states, and projective and anticipatory models of action.

The sense of agency that the organism feels when engaged in voluntary action is created by the correspondence between three kinds of neuronal signals: (1) somatosensory signals resulting directly from movement, (2) visual and auditory signals that may result indirectly from the organism's movements, and (3) the corollary discharge - the copy of the efferent motor command that generates the movement.

In sensorimotor loops, what is distinctive about the processes that specify the self as an agent, distinguishing between self and nonself, is that the sensory signals with an external

Fonseca, Isabel B; Fonseca, Jose B; Pereira, Vitor. (2013).

Beyond Embodiment: From Internal Representation of Action to Symbolic Processes. Liz Swan (ed.), Origins of Mind, Biosemiotics, Vol. 8 , 187-199.

https://doi.org/10.1007/978-94-007-5419-5 9

Springer, Dordrecht.

This manuscript version is made available under the CC-BY-NC-ND 4.0 license

http://creativecommons.org/licenses/by-nc-nd/4.0/ 
origin independent of the organism's own actions are noncontingent and uncorrelated with efferent action command signals; that is, a match between efferent and reafferent signals creates self-specifying meanings.

Dependent upon receptors and neuroanatomic pathways, reafference is distinguished from afferent signals in the process of comparing or matching these signals with efferent commands. The reafference is self-specifying because it is intrinsically related to a self-initiated action and it will originate reafferent signals that match the corollary discharge or efferent command. It is this correspondence between the efferent command signals and their reafferent consequences that signals that the information is self-specific and distinct from nonself-sensory afferent signals that are uncorrelated and noncontingent with efferent command.

\section{The Experiential Self, Interoceptive Loops, and Internal Cognitive Models}

Another kind of self-specifying processes can be found in the regulation of the organism's internal environment, in which loops of efferent-reafferent signals regulate the internal conditions for survival. In this case, efferent and afferent signals involve different structures from those related to voluntary action: brain stem nucleus and midbrain structures, somatoautonomic adjusting with low-level autonomic reflexes and high-level loops involving the lymbic structures, the hypothalamus, the insula, and the anterior cingulus. It is a homeostatic interoceptive system integrated in the vertical neuroaxis that specifies the state parameters of an experiential phenomenic self.

As opposed to the sensorimotor integration, which defines the relation between the organism and the external world, the homeostatic regulation specifies the organism's relation with its own environment and gives rise to subjective interoceptive feelings. The experience of feeling emerges from the binding of neuronal activities in a highly distributed system. Hypothetically, this subjective experience is related to a coherent matching between cognitive-affective states of higher-order, undifferentiated sensory signals processed by subcortical pathways involving the thalamus and limbic structures, and loops that regulate the organism's internal environment in structures such as the hypothalamus, insula, anterior cingulus, and other brain stem and midbrain structures, as well as low-level autonomic and somatic reflexes.

One method to study internal cognitive models of movement is based on the predictive effects of the sensory consequences of the subject's own actions. These effects consist in sensory suppression or attenuation of the reafferent signals and are produced in loops in which intentional commands modulate sensory feedback (Tsakiris and Hagaard 2005). The sensory suppression consists in the phenomenon of attenuating the reafferent sensory consequences of a self-generated movement. It has been thought that the reduction of sensory feedback of subject's own actions results from the voluntary nature of the movement. Numerous studies demonstrate the attenuation of the perceptual consequences of self-generated actions (Blakemore et al. 1999).

It is hypothesized that the perceptual consequences of self-generated actions are Fonseca, Isabel B; Fonseca, Jose B; Pereira, Vitor. (2013).

Beyond Embodiment: From Internal Representation of Action to Symbolic Processes. Liz Swan (ed.), Origins of Mind, Biosemiotics, Vol. 8 , 187-199.

https://doi.org/10.1007/978-94-007-5419-5_9

Springer, Dordrecht.

This manuscript version is made available under the CC-BY-NC-ND 4.0 license 
attenuated because internal models of the motor system use the efference copy (corollary discharge) to predict the consequences of the subject's own actions. This information is integrated in an internal "forward model" (Wolpert 1997) which is created and compares the predicted sensory outcome of the subject's own actions with the actual somatosensory reafferent feedback and other afferent messages that co-occur. The hypothesis of "efference copy" or copy of the motor command (Sperry (1950); Von Holst and Mittelstaedt 1950) was initially proposed to answer to Helmholtz's question: "How is it that, when we move our eyes, the world remains stable, despite the fact that the retinal image has moved?"

Von Holst and Mittelstaedt (1950) suggested that motor actions are accompanied by an efference copy of the action, which sends a "corollary discharge" to the sensory cortex signaling that impending signals are self-initiated or self-generated. The efference copy/corollary discharge mechanism works to suppress or reduce the perception of events that result from a self-generated action. Thus, it may allow an automatic distinction between internally and externally generated percepts. In the visual system, this system may serve to stabilize the visual image during eye movements, maintaining visuospatial constancy.

It is hypothesized that in sensory attenuation of the consequences of self-initiated actions, the process consists in analyzing a copy of an efferent motor command, an "efferenze copie" of a planned action, which is sent through a "feed-forward" mechanism to the appropriate sensory cortex, preparing it for the arrival of the feedback sensation-the efference copy works to suppress (or to reduce) perception when it results from a self-generated action.

These processes allow the organism to recognize that it has produced an action, and this information is used to modulate sensory consequences of movement. It is hypothesized that the prediction of sensory reafference and its integration in an internal model, relating the efferent, the afferent, and the behavioral intention, is expressed in a sensory suppression of inputs resulting from self-initiated actions.

\section{Development of Self-Awareness}

The consideration of these processes allows a hypothesis of defining the contribution of innate factors to the experience of self and finding indirect evidence about the way that meaning is influenced by innate factors linked to the structure and functions of the NS. By linking meaning and symbol formation to internal models of self-created in action planning and execution, it can be said that meaning and symbol formation originate from a sense of self as an agent.

There exists some evidence that the process of distinguishing the self-generated sensory reafferents from externally generated afferents, which indicate a sense of a proto-self and of agency, seems to begin early in life.

Fonseca, Isabel B; Fonseca, Jose B; Pereira, Vitor. (2013).

Beyond Embodiment: From Internal Representation of Action to Symbolic Processes. Liz Swan (ed.), Origins of Mind, Biosemiotics, Vol. 8, 187-199.

https://doi.org/10.1007/978-94-007-5419-5_9

Springer, Dordrecht.

This manuscript version is made available under the CC-BY-NC-ND 4.0 license

http://creativecommons.org/licenses/by-nc-nd/4.0/ 
Meltzoff and Moore (1977) describe imitative behavior in infants within 42 min of being bom; for example, babies imitate a tongue protrusion gesture performed by an adult. Meltzoff and Moore (1997, 1999) claim that perceivers, including infants, establish "supramodal representations" of bodily parts and their interrelations (in the case a tongue protruded between teeth) and, thus, that they have a type of proto-self or body schema that allows them to reproduce behaviors they observe. Other imitation behaviors, such as vocal imitation, observed in infants from 12 weeks of age are based essentially in intramodal comparisons (Kuhl and Melzoff 1996; Kuhl and Moore 1977).

In what concerns the distinction between sensory consequences of self-action and sensory consequences of stimuli independent of self-action, Rochat and Hespos (1997) observed that the rooting response of newborns (i.e., head orientation with mouth opening in the direction of a tactile stimulation on one of the cheeks) is significantly more frequent and predictable when the tactile stimulation comes from outside (single touch stimulation) than when results from spontaneous self-stimulation from the baby's own hand touching the cheek. This evidence of a differentiated rooting response in newborns suggests that they are capable of discriminating, at a very basic perceptual level, what corresponds to the sensory consequences of their own body movements from what corresponds to the external stimulation.

Developmental studies suggest that explicit self-awareness in infants comes much later. Between the 14th and 18th month, infants become embarrassed when they see in a mirror that there is a red spot on their face (Bertenthal and Fischer 1987). In this case, when children manifest shame or embarrassment, they take a meta-evaluative stance toward the embodied self. By the end of the second year, children begin to show self-consciousness - a meta-step in development that correlates with significant brain maturation, particularly in regions of the prefrontal cortex (Rochat 2010).

Nevertheless, manifestations of a self can be found in much earlier ages. There exists evidence that 4-month-olds start playing in front of mirrors (Tasakiris and Hagaard 2005) and are able to discriminate between their own and other's mirror images. Discrimination between self and others is interpreted as a proto form of self-awareness (Rochat and Striano 2002). The examples of imitative behavior or of distinct reactions to self and to external stimulation suggest that there exists a pre-reflexive form of experience of self, a proto-self, innate, present very early in ontogeny from birth, that allows a rudimentary distinction from self and nonself.

The existence of a proto-self in infants can further be conceived as a manifestation of an innate tendency to establish ties with a caregiver that will ensure safety, security, and protection. Meltzoff says that "we are bom social" - that is, there exists an attachment to a caring figure that ensures proximity between the infant and the attachment figure.

In what concerns meaning and proto-symbolic processes linked to action planning and anticipation integrated in the sense of self, these considerations point to some aspects that are innate and depend on the structure of the NS, which can be thought as structures of knowledge and meaning that are further elaborated in higher level semantic processes acquired during development in the interaction with the environment and also in linguistic processes.

Fonseca, Isabel B; Fonseca, Jose B; Pereira, Vitor. (2013).

Beyond Embodiment: From Internal Representation of Action to Symbolic Processes. Liz Swan (ed.), Origins of Mind, Biosemiotics, Vol. 8, 187-199.

https://doi.org/10.1007/978-94-007-5419-5 9

Springer, Dordrecht.

This manuscript version is made available under the CC-BY-NC-ND 4.0 license 


\section{What Are the Unique Characteristics of Self-Representation?}

The first and most primordial representation of the self is a body representation. The experience of the body has some characteristics that distinguish it from all other experiences, and it is the maximum invariant of the phenomenal and behavioral space.

The physiological sensory origin of this perceptual experience of body can be attributed to multiple sensory messages: pressure on and stretching of skin and deep tissues, friction and vibration on the skin, information about the body from neuromuscular and articulatory receptors, vestibular and balance information from the inner hear, the disposition and body volume from stretch receptors, nutrition and other homeostatic states from internal receptors, neuromuscular fatigue, and cerebral systems sensible to blood composition.

This systematization of somatic, interoceptive, and exteroceptive sensory systems shows that the body self doesn't rely on a single modality and neither is the information provided from a single modality. What distinguish the selfrepresentations from all other phenomenal representations is the unique representational structure in the brain that receives a permanent sensory input (Kinsboume 1995). What makes the body representation unique among all the percepts and phenomenal experiences is that the body representation is the maximum invariant - the center of the phenomenal space.

For all phenomena that can occur in consciousness, the body afferences are continuous and co-occurring permanently, some with a very slow or even nonexistent rate of sensory adaptation (such as proprioception, joint receptors, nociception). Although the relations in space and the movement can vary widely, the body remains a perceptual object that constantly generates afferent stimuli. Only the subject has first-person access to this ongoing sensory flux, which contributes to the subjective phenomenal experience of the self in a way that differs from the experience that results from an external object, which can be immediately socially shared (Zahavi 2002).

\section{Body Representation: The Integration Between Peripheral Sensory Stimulation and Central Neuronal Mapping in Somatic and Motor Cortex}

Peripheral sensory factors as well as central factors seem to play a role in the subjective feeling of embodiment. The consideration of some pathological conditions, such as "phantom phenomena," points to the contribution of central factors in body representation.

Having an experience of a part of the body that no longer exists, such as what occurs in phantom limb phenomena and phantom pain, has been attributed to a peripheral stimulation and also to a central factor. The body's inner representation 
at the neuronal level of the missing limb can be activated by intrinsic nervous activity or by activity that results from stimulation in other parts of the body. Whatever its origin, neuronal activity in the body's inner model (in cortical somatic maps) will be projected to the periphery that doesn't exist. The explanation of phantom limb phenomena depends on the activation of a central body neuronal model (Halligan 2002; Ramachandran and Hirstein 1998). This body model is innate but modified during development and later in adulthood by social interactions and behavioral interactions with the environment.

Other clinical observations of phantom limbs symptoms in $20 \%$ of children bom without one limb suggests that they develop a complex body model that includes the parts of the body that never existed (Ramachandran and Hirstein 1998). This phantom experience is attributed to a central origin and also suggests the existence of an innate body model or body schema.

\section{Heterogeneity of the Experiences of Self}

The self is the author, actor, and executor of its own actions; it acts and perceives from its own perspective. In this chapter, we have considered a sense of self that is related to the concept of body schema. Nevertheless, even within the sense of self as an agent, it is possible to distinguish different subjective experiences.

Although the sense of agency has been considered short-lived and phenomenologically recessive, the thin phenomenology of action has been analyzed. Pacherie $(2005,2008)$ identifies three cascading "stages" of action specification: F intentions (intentions directed to the future), $\mathrm{P}$ intentions (intentions directed to the present), and $\mathrm{M}$ intentions (motor intentions). For Pacherie, the sense of agency is complex and contains a variety of aspects: an experience of intentional causation, the sense of initiation, and the sense of control.

The $\mathrm{F}$ or future intentions are formed before the actions and represent the whole plan of actions. Their content is detached from the specific situation and therefore is conceptual and descriptive. The F intentions are means-end coherent, that is, consistent with the agent's beliefs and intentions.

The $\mathrm{P}$ intentions serve to implement action plans defined in $\mathrm{F}$ intentions. They anchor the action plan both in time and in the situation of action. They involve a transformation of the descriptive contents of the action plan into perceptual- movement contents constrained by the present spatial characteristics of the agent, the target of action, and the surrounding environment. The final stage is action specification which involves the transformation of the perceptual action contents of $\mathrm{P}$ intentions into sensorimotor representations ( $\mathrm{M}$ intentions) through a precise specification of the spatial and temporal characteristics of the constituent elements of the selected motor program (Pacherie 2007).

From a sense of agency, it is considered that the $\mathrm{F}$ - intentions that are relatively abstract and conceptual - may be spontaneously formulated and occur prior to the action. The $\mathrm{P}$ intention, which is more specific to the situation, occurs with higher temporal proximity to the action; involves a dynamic monitoring of the action; and can implement $\mathrm{F}$. The $\mathrm{P}$ intentions, which have an initiating function as they trigger the intended action and a 
sustaining function until completion of action, guide the function and monitor its effects. It can be supposed that each of these stages specifies a distinct agentive self-experience.

The neurophysiology of motor planning and regulation is well known within multiple neuronal systems. It seems possible to establish a parallelism between Pacherie's fine phenomenology of agency and the CNS's (central nervous system) hierarchical regulation of motor functions, and it should be noted that many functions of behavior planning, command, and execution operate at an unconscious level.

In the CNS, motor planning begins with a general outline of behavior and is translated into concrete motor responses through processing in the motor pathways. The regulation is hierarchical with levels of regulation (interdependent, parallel, with feed-forward and feedback neuronal loops): a superior level with functions in the definition of objectives or aims in behavior involving associative areas of the cortex and premotor cortex and interactions with basal ganglia; the next level associated with primary motor cortex of precentral gyrus and the cerebellum and with the function of specification of a motor program in which the kinetics and dynamics of movement is planned and commands issued; and an execution level involving brain stem nuclei and circuits of spinal cord, interneurons, and motor neurons that regulate a variety of automated movements that control posture and locomotion (Kandel 2000).

\section{Beyond Embodiment: Internal Representation of the Model of Action}

In sensorimotor integration, representation is tentatively defined as a form of intentional internal modeling. This internal modeling is invoked to explain the fact that behavior is oriented to the achievement of future goals and is relatively independent from immediate environmental stimuli or specific sensorimotor representations. Internal modeling occurs in a projective and anticipatory way, and what is represented are anticipated states or intentional goals in an abstract form.

One of the most fundamental properties of cognition is, as Kenneth Craik put it, its power to predict events (Craik 1943).

In representations, there are three essential processes: (1) translation of external processes and internal data into words, numbers, or other symbols; (2) emergence of other symbols by a process of reasoning, deduction, and inferences, that is, the process of prediction; and (3) retranslation of these symbols into external processes - or at least a correlation between these symbols and external events (as in realizing that a prediction is fulfilled), the result of which is translated into the world.

The process of reasoning produces a final result similar to that which might have been reached by causing the actual physical process to occur. The thought processes have homomorphic properties with external events and so can be used to predict these external events (on the condition, there is a time delay between the two).

Thus according to Craik, the essence of thought is to provide a model of the external 
world. The mental prediction (or anticipatory model) in internal modeling is flexible and versatile — sensing, modeling, planning, and acting.

To invoke Liz Swan and Louis Goldberg (2010a) about symbols such as words, icons, or signals, symbols are elements that map signifiers to that which they signify. These mappings can be either arbitrary or transparent. Words are signifiers arbitrarily related to a significant; an icon is a transparent signifier that is linked by resemblance to the things they refer to, and signals are transparent signifiers that have a physical or mechanical connection to other objects.

The model they propose is one wherein symbol formation has a sensory- perceptive origin, that is, sensory receptors detect the presence of and respond to stimuli, which are processed and coded by perceptual symbol formation. The symbols induce effector processes (Swan and Goldberg 2010a, b).

In this chapter, we have tried to link symbol formation to a sense of self that invokes meaning and symbolic processes that occur in a nonconscious proto-self that constitutes a first-order representation. The second-order representation includes the relation between the self and the object. The third-order representation involves meta-representation of autoreflexive processes.

The model we propose takes an efferent-anticipatory point of view in which symbolic meanings are created in an interactive internal space, referred to as the agent or self, that is, an internal model that binds perceptual present, past memories, and also future desirable states. It is proposed that symbols are projective, anticipatory, or beyond immediate instantiations. They are abstract and intentional, and in this sense, symbols are beyond embodiment.

\section{Conclusion}

We have proposed an individual-centered perspective for symbolization and meaning processes. The embodied ground of meaning, linked to the formation of an internal model, allows phenomenic agentive first-person experience and shapes judgments. Meaning arises in this internal model, which integrates external and internal influences and recruits neural systems involved in perception, movement, and emotion. The embodied model of symbolization processes points to mental simulation, anticipatorily driven by a complex interplay between sensory and motor components, in which intentions and future aims or desirable states are represented. These successively more complex and higher-order behavioral strategies are recursively generated independent of their embodiment. 


\section{References}

Bertenthal, B., \& Fischer, K. (1987). Development of self recognition in the infant. Developmental Psychology, 14,44-50.

Blakemore, S.-J., Frith, C. D., \& Wolpert, D. (1999). Spatio-temporal prediction modulates the perception of self produced stimuli. Journal of Cognitive Neuroscience, 11, 551-559.

Craik, K. (1943). The nature of explanation. Cambridge: Cambridge University Press. Gallagher, S. (2000).

Philosophical conceptions of the self: Implications for cognitive science. Trends in Cognitive Science, 4(1), $14-21$.

Gallagher, S. (2012). Multiple aspects in the sense of agency. New Ideas in Psychology, 30(1), 15-31.

Halligan, P. W. (2002). Phantom limbs: The body in the mind. Cognitive Neuropsychiatry, 7(3), 252-268.

Kandel, E. R. (2000). From nerve cells to cognition: The internal cellular representation required for perception and action. In E. R. Kandel, J. H. Schawrtz, \& T. M. Jessell (Eds.), Principles of neural science (pp. 381-402). New York: McGraw Hill.

Kinsboume, M. (1995). Awareness of one's own body: An attentional theory of its nature, development and brain basis. In J. L. Bermudez, A. Marcel, \& N. Eilan (Eds.), The body and the self(pp. 205-223). Cambridge, MA: MIT Press.

Kuhl, P., \& Moore, M. (1977). Infant vocalizations in response to speech: Vocal imitation and developmental change. The Journal of the Acoustical Society of America, 100, 2425-2438.

Kuhl, P. H., \& Melzoff, A. N. (1996). Infant vocalizations in response to speech: Vocal imitation and developmental change. Journal of the Acoustic Society, 100, 2425-2438.

Llinas, R. R. (1987). 'Mindness' as a functional state of the brain. In C. Blakemore \& S. Greenfield (Eds.), Mindwaves. Thoughts on intelligence, identity and consciousness. New York: Basil Blackwell.

Llinas, R. R. (2001). I of the vortex. From neurons to self. Cambridge, MA: MIT Press. Meltzoff, A., \&

Moore, M. K. (1997). Explaining facial imitation: A theoretical model. Early Development and Parenting, 6,179-192.

Meltzoff, A., \& Moore, K. (1999). Persons and representation: why infant imitation is important for theories of human development. In J. Nadel \& B. Butterworth (Eds.), Imitation in infancy (pp. 9-35). Cambridge: Cambridge University Press.

Pacherie, E. (2005). Perceiving intentions. In J. Saagua (Ed.), A explicagao da intrerpretagao humana (pp. 401-414). Lisbon: Edi^oes Colibri.

Pacherie, E. (2007). The sense of control and the sense of agency. Psyche, 73(1), 1-30. Pacherie, E. (2008).

The phenomenology of action: A conceptual framework. Cognition, 107, 179-217.

Ramachandran, V. S., \& Hirstein, W. (1998). The perception of phantom limbs. Brain, 121, 1603-1630.

Rochat, P. (2010). The innate sense of the body develops to become a public affair by 2-3 years. Neuropsychologia, 48,738-745.

Rochat, P, \& Hespos, S. J. (1997). Differential rooting response by neonates: Evidence for an early sense of self. Early development and Parenting, 6, 105-112.

Rochat, P., \& Striano, T. (2002). Who's in the mirror? Self-other discrimination in specular images by fourand nine-month- old infants. Child Development, 73,35-46.

Sperry, R. W. (1950). Neural basis of the spontaneous optokinetic response produced by visual inversion. Journal of Comparative and Physiological Psychology.

Swan, L. S., \& Goldberg, L. J. (2010a). Biosymbols: Symbols in fife and mind. Biosemiotics, 3(1), 17-31.

Swan, L. S., \& Goldberg, L. J. (2010b). How is meaning grounded in the organism? Biosemiotics, 3(2), 131-146.

Beyond Embodiment: From Internal Representation of Action to Symbolic Processes

Tsakiris, M., \& Haggard, P. (2005). Experimenting with the acting self. Cognitive Neuropsychology,

Fonseca, Isabel B; Fonseca, Jose B; Pereira, Vitor. (2013). 
$22(3 / 4), 387^{\wedge} 107$.

Von Holst, E., \& Mittelstaedt, H. (1950). Das Reaffemzprinzip echselwirkungen zwichen zentrainervensystem und peripherie. Naturwissenschalten, 37,464-476.

Wolpert, D. M. (1997). Computational approaches to motor control. Trends in Cognitive Sciences, 1,209-216.

Zahavi, D. (2002). First-person thoughts and embodied self-awareness. Phenomenology and the Cognitive Sciences, 1, 7-26. 